\title{
Simple closed geodesics on regular tetrahedra in Lobachevsky space
}

\author{
Alexander A. Borisenko, Darya D. Sukhorebska
}

Abstract. We obtained a complete classification of simple closed geodesics on regular tetrahedra in Lobachevsky space. Also, we evaluated the number of simple closed geodesics of length not greater than $L$ and found the asymptotic of this number as $L$ goes to infinity.

Keywords: closed geodesics, simple geodesics, regular tetrahedron, Lobachevsky space, hyperbolic space. $M S C$ : $53 \mathrm{C} 22,52 \mathrm{~B} 10$

\section{Introduction}

A closed geodesic is called simple if this geodesic is not self-intersecting and does not go along itself. In 1905 Poincare proposed the conjecture on the existence of three simple closed geodesics on a smooth convex surface in three-dimensional Euclidean space. In 1917 J. Birkhoff proved that there exists at least one simple closed geodesic on a Riemannian manifold that is homeomorphic to a sphere of arbitrary dimension [1]. In 1929 L. Lyusternik and L. Shnirelman obtained that there exist at least three simple closed geodesics on a compact simply-connected two-dimensional Riemannian manifold [2], 3]. But the proof by Lyusternik and Shnirelman contains substantial gaps. I. A. Taimanov gives a complete proof of the theorem that on each smooth Riemannian manifold homeomorphic to the two-dimentional sphere there exist at least three distinct simple closed geodesics [4].

In 1951 L. Lyusternik and A. Fet stated that there exists at least one closed geodesic on any compact Riemannian manifold [5]. In 1965 Fet improved this results. He proved that there exist at least two closed geodesics on a compact Riemannian manifold under the assumption that all closed geodesics are non-degenerate [6]. V. Klingenberg generalized this theorem. He showed that on compact Riemannian manifolds with a finite fundamental group there exist infinitely many closed geodesics [7].

Due to works of Huber [8], 9] it is known that on complete closed two-dimensional surfaces of constant negative curvature the number of closed geodesics of length not greater than $L$ is of order $e^{L} / L$ as $L \rightarrow+\infty$. Ya. Sinai [10] and G. Margulis [11] and others generalized this estimation for compact $n$-dimensional manifolds of non-constant negative curvature. Igor Rivin studied the growth of $N(L)$, the number of simple closed geodesics of length bounded above by $L$ on a hyperbolic surface of genus $g$ with $n$ points at infinity. He proved [12] that there exist constants $c_{1}$ and $c_{2}$ such that

$$
c_{1} L^{6 g-6+2 n} \leq N(L) \leq c_{2} L^{6 g-6+2 n} .
$$

M. Mirzakhani [13] showed that the constants $c_{1}$ and $c_{2}$ are equal and found its value.

Geodesics on the non-smooth surfaces are also investigated. A. Cotton and others described all simple closed geodesics on a cube, regular tetrahedron, regular octahedron and rectangular prism [14]. K. Lawson and others obtain a complete classification of simple closed geodesics on eight convex deltahedra such that the faces of these deltahedra are regular triangles [15]. 
D. Fuchs and K. Fuchs supplemented and systematized results about closed geodesics on a regular polyhedra in three-dimensional Euclidean space [16], [17. V. Protasov obtained conditions for the existence of closed geodesics on an arbitrary simplex and gave the estimate for the number of closed geodesics on a simplex depending on the largest deviation from $\pi$ of the sum of the plane angles at a vertex of the simplex [18].

The aim of this paper is to describe all simple closed geodesics on regular tetrahedra in three-dimensional Lobachevsky space. In Euclidean space since the Gaussian curvature of faces of a tetrahedron is equal to zero, it follows that the curvature of the tetrahedron is concentrated into its vertices. In Lobachevsky space the faces of a tetrahedron have a constant negative curvature. Thus the curvature of such tetrahedron defined not only by its vertices but also by its faces. Furthermore, all regular tetrahedra in Euclidean space are homothetic to each others. In Lobachevsky space the intrinsic geometry of a regular tetrahedron depends on the plane angles of the faces of this tetrahedron. Regular euclidean triangles form a regular tiling of the euclidean plane. From this it is easy to proof the full classification of closed geodesics on regular tetrahedrons in Euclidean space. In general it is impossible to make a triangular tiling of the Lobachevsky plane by regular triangles.

It is true

Theorem 1. On a regular tetrahedron in Lobachevsky space for any coprime integers $(p, q)$, $0 \leq p<q$, there exists unique, up to the rigid motion of the tetrahedron, simple closed geodesic of type $(p, q)$. The geodesics of type $(p, q)$ exhaust all simple closed geodesics on a regular tetrahedron in Lobachevsky space.

The simple closed geodesic of type $(p, q)$ has $p$ points on each of two opposite edges of the tetrahedron, $q$ points on each of another two opposite edges, and there are $(p+q)$ points on each edges of the third pair of opposite edges.

Theorem 2. Let $N(L, \alpha)$ be a number of simple closed geodesics of length not greater than $L$ on a regular tetraedron with plane angles of the faces equal to $\alpha$ in Lobachevsky space. Then there exists a function $c(\alpha)$ such that

$$
N(L, \alpha)=c(\alpha) L^{2}+O(L \ln L)
$$

where $O(L \ln L) \leq C L \ln L$ as $L \rightarrow+\infty, c(\alpha)>0$ when $0<\alpha<\frac{\pi}{3}$ and

$$
\lim _{\alpha \rightarrow \frac{\pi}{3}} c(\alpha)=+\infty ; \quad \lim _{\alpha \rightarrow 0} c(\alpha)=\frac{27}{32(\ln 3)^{2} \pi^{2}}
$$

\section{Definitions}

A curve $\gamma$ is called a geodesic if any sufficiently small subarc of $\gamma$ realizes a shortest path between endpoints of this subarc. On a convex polyhedron a geodesic satisfies the following properties [19]:

1) within every faces the geodesic is a straight line segment;

2) the geodesic traverses an interior point of an edge "without refraction", i.e., the angles that it forms with the edge are equal from the two sides;

3) the geodesic does not pass through a vertex of a convex polyhedron.

Remark 1. By a straight line segment we mean a segment of a geodesic in the space of constant curvature.

Assume a geodesic on a convex polyhedron starts at a point $X$ of an edge. Then it goes into a face of the polyhedron, intersects another edge of the same face at a point $Y$ and then 
passes to the next face, and so on. Draw these faces in the plane along the geodesic. In this way we obtain the polygon that is called the development of a polyhedron along the geodesic. The geodesic becomes a straight line on it. If a geodesic is closed, then this straight line arrives to the initial edge at the point $X^{\prime}$ at the same position as $X$.

We assume that the Gaussian curvature of Lobachevsky space (hyperbolic space) is equal to -1 . By a regular tetrahedron in three-dimensional Lobachevsky space we understand a closed convex polyhedron formed by four regular triangles. These triangles are called faces and they form regular trihedral angles in the vertices of the tetrahedron. Note that the plane angle $\alpha$ of the face satisfies the inequality $0<\alpha<\frac{\pi}{3}$. Up to a rigid motion in Lobachevsky space there exists a unique tetrahedron with a given plane angle of its face. The length $a$ of every its edge is equal to

$$
a=\operatorname{arcosh}\left(\frac{\cos \alpha}{1-\cos \alpha}\right) .
$$

The following auxiliary formulas follows from (2.1):

$$
\begin{gathered}
\tanh a=\frac{\sqrt{2 \cos \alpha-1}}{\cos \alpha} ; \\
\cosh \frac{a}{2}=\frac{1}{2 \sin \frac{\alpha}{2}} ; \\
\sinh \frac{a}{2}=\frac{\sqrt{2 \cos \alpha-1}}{2 \sin \frac{\alpha}{2}} .
\end{gathered}
$$

Consider the Cayley-Klein model of hyperbolic space. In this model points are represented by the points in the interior of the unit ball [21]. Geodesics in this model are the chords of the ball. Assume that the center of the circumscribed sphere of a regular tetrahedron coincides with the center of the model. Then the regular tetrahedron in Lobachevsky space is represented by a regular tetrahedron in Euclidean space.

\section{Necessary conditions for the simplicity of a geodesic on a regular tetrahedron in Lobachevsky space}

We start this section with formulation some useful lemmas.

Lemma 3.1. 20] . Let $M$ be a complete simply connected Riemannian manifold of nonpositive curvature. Let $\gamma_{1}(s):[0,1] \rightarrow M$ and $\gamma_{2}(s):[0,1] \rightarrow M$ be geodesics. Let $\sigma_{s}(t):[0,1] \rightarrow M$ be a geodesic such that $\sigma_{s}(0)=\gamma_{1}(s), \sigma_{s}(1)=\gamma_{2}(s)$, and let $\rho(s)$ be the length of $\sigma_{s}$. Then $\rho(s)$ is a convex function.

Corollary 3.1. [20]. Let $\gamma(s)$ be a geodesic in a complete simply connected Riemannian manifold $M$ of nonpositive curvature. Then the function $\rho(s)$ of a distance from the fixed point $p$ on $M$ to the points on $\gamma(s)$ is a convex function.

Lemma 3.2. [22]. Let $A B C$ be a right triangle $\left(\angle C=\frac{\pi}{2}\right)$ in Lobachevsky space. Then

$$
\begin{aligned}
\cosh |A B|=\cosh |C A| \cosh |C B| & \text { (the hyperbolic Pythagorean theorem); } \\
& \tanh |A C|=\tanh |A B| \cos \angle A ; \\
& \tanh |C B|=\sinh |A C| \tan \angle A ; \\
& \sinh |C B|=\sinh |A B| \tan \angle A .
\end{aligned}
$$


Consider a regular tetrahedron with the plane angle $\alpha$ of its face. Denote by $h$ the altitude of its face. Using (3.2) and 2.2), we get

$$
\tanh h=\tanh a \cos \frac{\alpha}{2}=\cos \frac{\alpha}{2} \frac{\sqrt{2 \cos \alpha-1}}{\cos \alpha} .
$$

Lemma 3.3. If a geodesic successively intersects three edges sharing a common vertex on a regular tetrahedron in Lobachevsky space and one of these edges twice, then this geodesic is self-intersecting.

Proof. Let $A_{1} A_{2} A_{3} A_{4}$ be a regular tetrahedron in Lobachevsky space. Suppose the geodesic $\gamma$ successively intersects $A_{4} A_{1}, A_{4} A_{2}$ and $A_{4} A_{3}$ at the points $X_{1}, X_{2}, X_{3}$ and then intersects $A_{4} A_{1}$ at the point $Y_{1}$. If $Y_{1}$ coincides with $X_{1}$, then this point is a self-intersection point of $\gamma$. Suppose that the length of $A_{4} X_{1}$ is less than the length of $A_{4} Y_{1}$.

Consider the trihedral angle at the vertex $A_{4}$. Cut this angle along the edge $A_{4} A_{1}$ and develop it to the plane. We obtain the convex polygon $A_{1} A_{4} A_{1}^{\prime} A_{3} A_{2}$. The part $X_{1} X_{2} X_{3} Y_{1}$ of the geodesic is the straight line segment on the development.

Let $\rho\left(A_{4}, X\right)$ be the distance function between the vertex $A_{4}$ and a point $X$ on $\gamma$. From Corollary 3.1 it follows that the function $\rho\left(A_{4}, X\right)$ is a convex function. This function attains its minimum at the point $H_{0}$ such that $A_{4} H_{0}$ is orthogonal to $\gamma$ and $\angle H_{0} A_{4} Y_{1}>\frac{3 \alpha}{2}$.

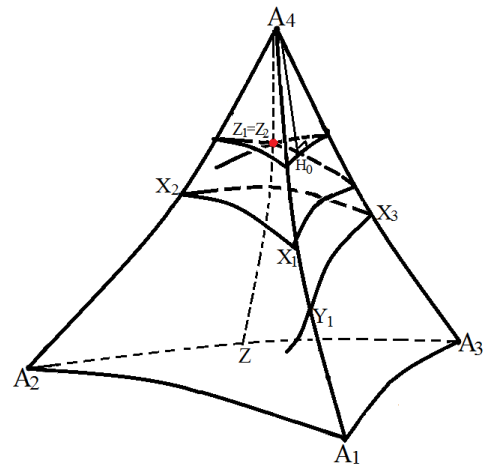

Figure 1

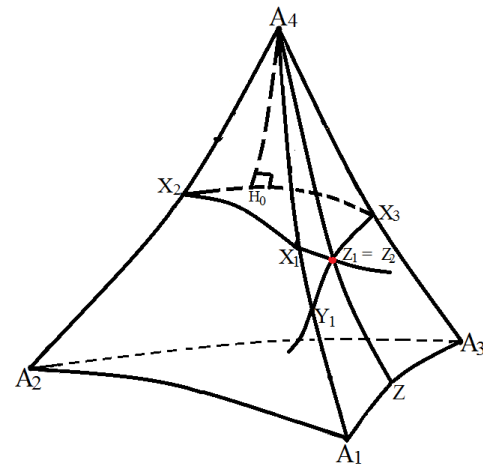

Figure 2

On the segment $H_{0} Y_{1}$ mark the point $Z_{1}$ such that $\angle H_{0} A_{4} Z_{1}=\frac{3 \alpha}{2}$. Then on the part of $\gamma$ started from the point $H_{0}$ in the direction opposite to $H_{0} Y_{1}$ mark the point $Z_{2}$ such that $\angle H_{0} A_{4} Z_{2}=\frac{3 \alpha}{2}$. The point $Z_{2}$ also lies in a face incident to the vertex $A_{4}$.

Since $\angle H_{0} A_{4} Z_{1}=\angle H_{0} A_{4} Z_{2}=\frac{3 \alpha}{2}$, it follows that the segments $A_{4} Z_{1}$ and $A_{4} Z_{2}$ belong to the segment $A_{4} Z$ opposite to $A_{4} H_{0}$ on the tetrahedron. Furthermore, the triangles $Z_{1} A_{4} H_{0}$ and $Z_{2} A_{4} H_{0}$ are equal. It follows that on the tetrahedron the points $Z_{1}$ and $Z_{2}$ correspond to the same point on $A_{4} Z$. This point is the self-intersection point of the geodesic $\gamma$ (Figure 1 . Figure 21.

Lemma 3.4. Let $d$ be the smallest distance between the set of vertices of a regular tetrahedron in Lobachevsky space and a simple closed geodesic on this tetrahedron. Then

$$
\tanh d>\cos \frac{3 \alpha}{2} \cos \frac{\alpha}{2} \frac{\sqrt{2 \cos \alpha-1}}{\cos \alpha}
$$

where $\alpha$ is the plane angle of a face of the tetrahedron.

Proof. Consider a regular tetrahedron $A_{1} A_{2} A_{4} A_{3}$ in Lobachevsky space and a simple closed geodesic $\gamma$ on this tetrahedron. Assume that the distance between vertex $A_{4}$ and $\gamma$ is the 


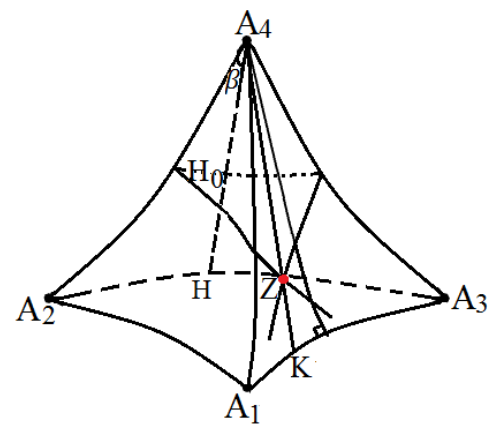

Figure 3

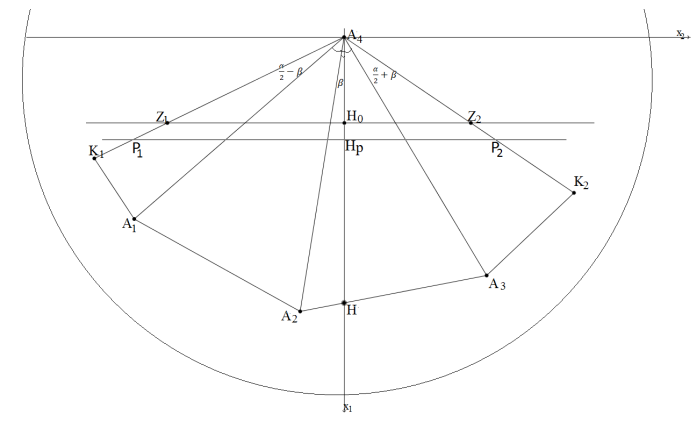

Figure 4

smallest distance from the set of vertices of a tetrahedron to $\gamma$. Construct the line segment $A_{4} H$ that is orthogonal to $\gamma$ at the point $H_{0}$. Assume $A_{4} H$ belongs to the face $A_{2} A_{4} A_{3}$. Denote by $\beta$ the angle between $A_{4} H$ and $A_{4} A_{2}$. Without loss of generality we assume that $0 \leq \beta \leq \frac{\alpha}{2}$.

Construct the segment $A_{4} K$ such that the plane angle between $A_{4} K$ and $A_{4} H$ equals $\frac{3 \alpha}{2}$ (Figure 3). It follows that $A_{4} K$ lies in the face $A_{1} A_{4} A_{3}$ and the angle between $A_{4} K$ and $A_{4} A_{1}$ equals $\left(\frac{\alpha}{2}-\beta\right)$ on this face. Note that if $\beta=\frac{\alpha}{2}$, then $A_{4} K$ coincides with $A_{4} A_{1}$. If $\beta=0$, then $A_{4} K$ coincides with the altitude of the face and has the smallest length.

Cut the trihedral angle at the vertex $A_{4}$ by the line $A_{4} K$ and develop it to the plane. We obtain the convex polygon $K_{1} A_{4} K_{2} A_{3} A_{2} A_{1}$. Consider the Cayley-Klein model of the hyperbolic plane. Assume that the vertex $A_{4}$ of the development coincide with the centre of the model (Figure 4). The angle $K_{1} A_{4} K_{2}$ equals $3 \alpha$. The segment $A_{4} H$ corresponds to the bisector of the angle $K_{1} A_{4} K_{2}$. The geodesic $\gamma$ is a straight line that is ortogonal to $A_{4} H$ at the point $H_{0}$.

On the lines $A_{4} K_{1}$ and $A_{4} K_{2}$ mark the points $P_{1}$ and $P_{2}$ respectively so that the length of $A_{4} P_{1}$ and the length of $A_{4} P_{2}$ are equal to the length of the altitude $h$ of the face (Figure 4 ). The line segment $P_{1} P_{2}$ is ortogonal to $A_{4} H$ at the point $H_{p}$. From equation (3.2) applied to the triangle $A_{4} H_{p} P_{1}$ we obtain

$$
\tanh \left|A_{4} H_{p}\right|=\cos \frac{3 \alpha}{2} \tanh h
$$

If $d \leq\left|A_{4} H_{p}\right|$, then $\gamma$ lies above the segment $P_{1} P_{2}$. It follows that $\gamma$ intersects the lines $A_{4} K_{1}$ and $A_{4} K_{2}$ at the points $Z_{1}$ and $Z_{2}$ respectively.

The segments $A_{4} K_{1}$ and $A_{4} K_{2}$ correspond to the segment $A_{4} K$ on the tetrahedron. Then the points $Z_{1}^{\prime}$ and $Z_{2}^{\prime}$ are the same point $Z$ on the segment $A_{4} K$. We obtain that the point $Z$ is the self-intersection point of the geodesic $\gamma$.

Thus we get that $d>\left|A_{4} H_{p}\right|$ is a necessary condition for the geodesic $\gamma$ to have no points of self-intersection on the regular tetrahedron in Lobachevsky space. From (3.7) it follows that

$$
\tanh d>\cos \frac{3 \alpha}{2} \tanh h .
$$

Using (3.5) and (3.8), we obtain inequality 3.6.

Corollary 3.2. Let d be the smallest distance between the set of vertices of a regular tetrahedron in Lobachevsky space and a simple closed geodesic on this tetrahedron. Then

$$
d>\frac{1}{2} \ln \left(\frac{\sqrt{2 \pi^{3}}+(\pi-3 \alpha)^{\frac{3}{2}}}{\sqrt{2 \pi^{3}}-(\pi-3 \alpha)^{\frac{3}{2}}}\right),
$$

where $\alpha$ is the plane angle of a face of the tetrahedron. 
Proof. It is known that

$$
\sin y>\frac{2}{\pi} y \text { for } 0<y<\frac{\pi}{2} .
$$

Consider the function $\sqrt{2 \cos \alpha-1}$. We get

$$
2 \cos \alpha-1=2 \cos \alpha-2 \cos \frac{\pi}{3}=4 \sin \left(\frac{\pi}{6}-\frac{\alpha}{2}\right) \sin \left(\frac{\pi}{6}+\frac{\alpha}{2}\right) .
$$

On the interval $\left(0, \frac{\pi}{3}\right)$ the function $\sin \left(\frac{\pi}{6}+\frac{\alpha}{2}\right)$ increases. At $\alpha=0$ this function equals $\frac{1}{2}$. It follows that $\sin \left(\frac{\pi}{6}+\frac{\alpha}{2}\right)>\frac{1}{2}$ when $\alpha \in\left(0, \frac{\pi}{3}\right)$. The function $\sin \left(\frac{\pi}{6}-\frac{\alpha}{2}\right)$ increases on the interval $\left(0, \frac{\pi}{3}\right)$. From 3.10 we get $\sin \left(\frac{\pi}{6}-\frac{\alpha}{2}\right)>\frac{1}{\pi}\left(\frac{\pi}{3}-\alpha\right)$. We obtain

$$
\sqrt{2 \cos \alpha-1}>\sqrt{\frac{2}{3 \pi}(\pi-3 \alpha)}
$$

Consider $\cos \frac{3 \alpha}{2}$. Since $0<\alpha<\frac{\pi}{3}$, it follows that $0<\frac{3 \alpha}{2}<\frac{\pi}{2}$. On the interval $0<y<\frac{\pi}{2}$ the function $\cos y$ decreases. We have the estimate

$$
\cos y>1-\frac{2}{\pi} y, \quad 0<y<\frac{\pi}{2}
$$

From this estimate we obtain

$$
\cos \frac{3 \alpha}{2}>\frac{1}{\pi}(\pi-3 \alpha) .
$$

On the interval $0<\alpha<\frac{\pi}{3}$ the function $\cos \frac{\alpha}{2}$ decreases. We have $\cos \frac{\alpha}{2}>\frac{\sqrt{3}}{2}$ when $0<\alpha<\frac{\pi}{3}$.

From the computation above and (3.11), (3.12) it follows that

$$
\tanh d>\frac{1}{\sqrt{2 \pi^{3}}}(\pi-3 \alpha)^{\frac{3}{2}} .
$$

From inequality $(3.13)$ we obtain inequality $(3.9)$.

\section{Properties of closed geodesics on regular tetrahedra in Euclidean space}

Consider a regular tetrahedron $A_{1} A_{2} A_{3} A_{4}$ in Euclidean space (Figure 5). Assume the geodesic $\gamma$ starts at the point $X$ in the edge $A_{1} A_{2}$ and then goes to the face $A_{1} A_{2} A_{4}$. The development of a regular tetrahedron along the geodesic is a part of the regular triangular tiling of the Euclidean plane. Label the vertices of this tiling according to the tetrahedron's vertices. It other words, for any development of the tetrahedron the labeling of its vertices corresponds to the labeling of the vertices of the tiling (Figure 6).

To construct a closed geodesic, it is sufficient to choose two identically oriented edges in the tiling, for example $A_{1} A_{2}$, not on the same line, to mark points $X$ and $X^{\prime}$ on these edges at the same distance from $A_{1}$ and to join $X$ and $X^{\prime}$ by the line segment. Note that the points $X$ and $X^{\prime}$ are such that the line $X X^{\prime}$ does not pass through any vertex of the tiling. The line segment $X X^{\prime}$ realizes a closed geodesic on the tetrahedron, and every closed geodesic can be obtained in this way [16].

Since the segments of the geodesic within every face are parallel to each other, it follows that all closed geodesic on a regular tetrahedron are non-self-intersecting (Figure 5).

Two geodesics are called equivalent if they pass through the same edges in the same order on the polyhedron. 


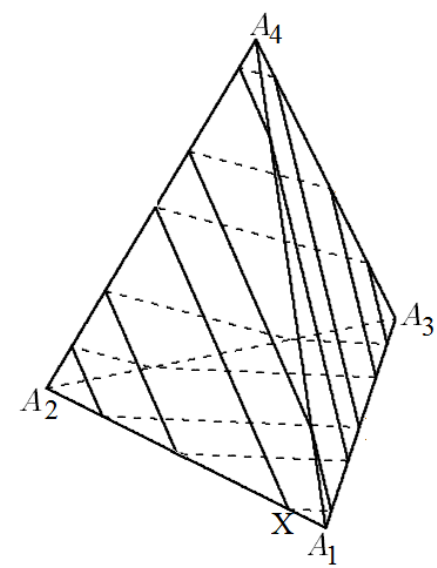

Figure 5

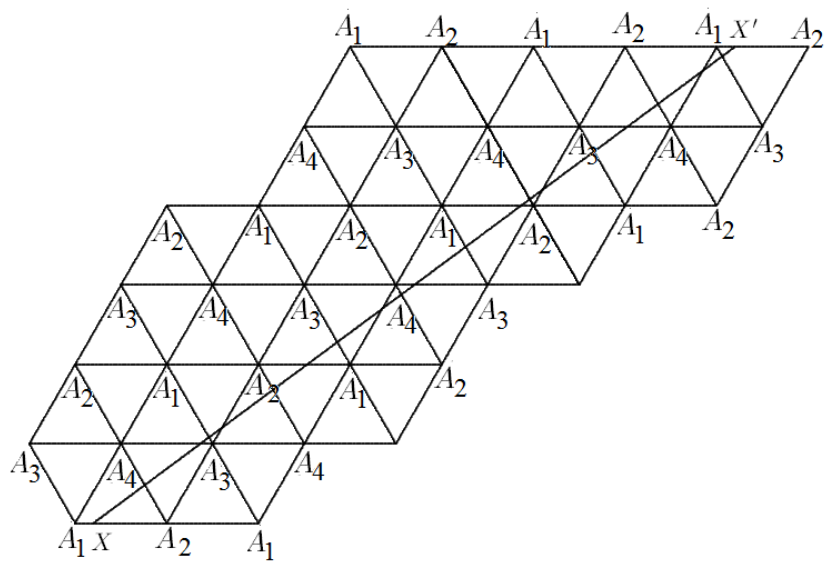

Figure 6

Lemma 4.1. Let $\gamma$ be a simple closed geodesic on a regular tetrahedron in Euclidean space. Then there exists a simple closed geodesic $\gamma_{0}$ equivalent to $\gamma$ such that $\gamma_{0}$ passes through the midpoints of two pairs of opposite edges on the tetrahedron.

Proof. Consider a triangular tiling of the Euclidean plane and label the vertices of the tilling according to the vertices of a regular tetrahedron (Figure 6). Assume that the Cartesian coordinate system has the origin at the vertex $A_{1}$ and the $x$-axis is going along the edge $A_{1} A_{2}$. Denote by $\left(x_{v}, y_{v}\right)$ the coordinates of the vertices. The vertices $A_{1}$ and $A_{2}$ belong to the line $y_{v}=2 k \frac{\sqrt{3}}{2}$, and their first coordinate is $x_{v}=l(k, l \in \mathbb{Z})$. The vertices $A_{3}$ and $A_{4}$ belong to the line $y_{v}=(2 k+1) \frac{\sqrt{3}}{2}$, and their first coordinate is $x_{v}=l+\frac{1}{2}(k, l \in \mathbb{Z})$.

Suppose a simple closed geodesic $\gamma$ starts at the point $X$ with coordinates $(\mu, 0)$, where $0<\mu<1$. Then the endpoint $X^{\prime}$ of $\gamma$ has the coordinates $(\mu+q+2 p, q \sqrt{3})$, where $p, q$ are nonnegative integer numbers. The line $X X^{\prime}$ is $y=\frac{q \sqrt{3}}{q+2 p}(x-\mu)$. Note that if $p, q$ are coprime integers, then the geodesic does not go along itself [16].

First we proof that if geodesic passes through the midpoint of the one edge, then it passes through the midpoints of two pairs of the opposite edges. Assume that a closed geodesic $\gamma_{0}$ passes through the midpoint of the edge $A_{1} A_{2}$. Then the equation of $\gamma_{0}$ is

$$
y=\frac{q \sqrt{3}}{q+2 p}\left(x-\frac{1}{2}\right) .
$$

Substituting the coordinates of the points $A_{3}$ and $A_{4}$ to equation (4.1), we get

$$
q(2 l-2 k-1)=2 p(2 k+1) .
$$

If $q$ is even then there exist $k$ and $l$ satisfying equation (4.2). It follows that there exist the vertex of the tiling such that $\gamma_{0}$ passes through this vertex. It contradicts the properties of $\gamma_{0}$, therefore $q$ is an odd integer.

The points $X_{1}$ and $X_{1}^{\prime}$ with coordinates $\left(\frac{1}{2}, 0\right)$ and $\left(q+2 p+\frac{1}{2}, q \sqrt{3}\right)$ satisfy equation 4.1 . These points are the middle point of the edge $A_{1} A_{2}$ on the tetrahedron. Suppose that the point $X_{2}$ is the midpoint of $X_{1} X_{1}^{\prime}$. Then the coordinates of $X_{2}$ are $\left(\frac{q}{2}+p+\frac{1}{2}, \frac{q}{2} \sqrt{3}\right)$. Substituting $q=2 k+1$, we obtain $X_{2}=\left(k+p+1,\left(k+\frac{1}{2}\right) \sqrt{3}\right)$. Since the second coordinate of $X_{2}$ is $\left(k+\frac{1}{2}\right) \sqrt{3}$, where $k$ is integer, then the point $X_{2}$ belongs to the line, that contains the vertices $A_{3}$ and $A_{4}$. Since the first coordinate of $X_{2}$ is an integer, it follows that $X_{2}$ is situated in the center of the edge $A_{3} A_{4}$. 


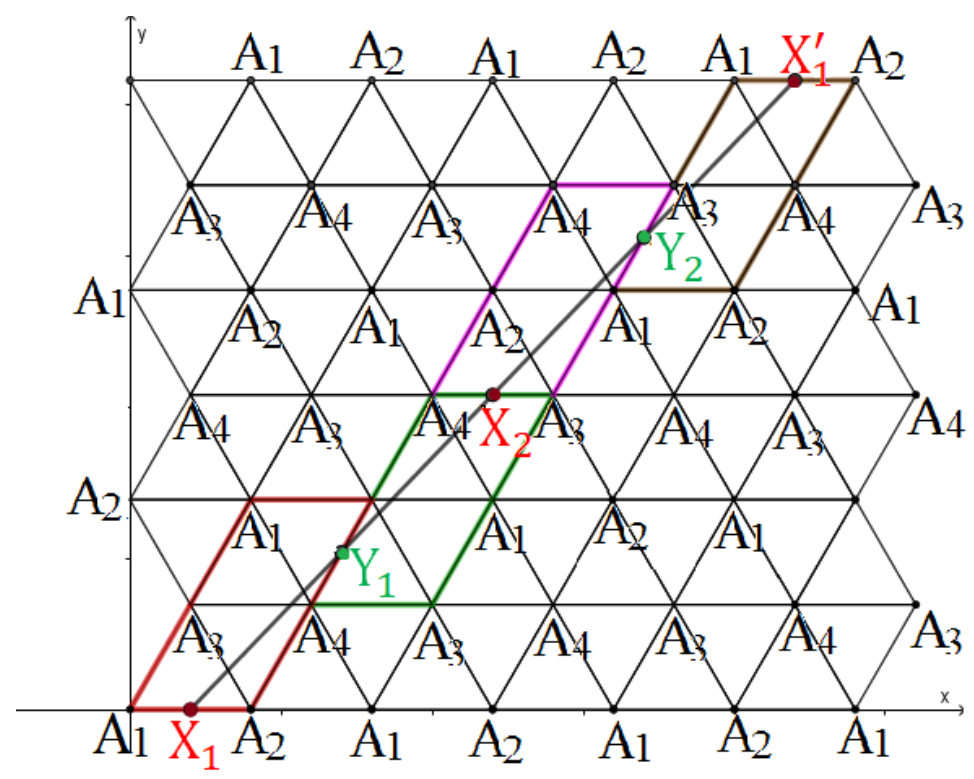

Figure 7

Now we prove that if a closed geodesic on a tetrahedron passes through the midpoint of one edge, then it passes through the midpoint of the opposite edge.

Let $Y_{1}$ be the midpoint of $X_{1} X_{2}$. Then the coordinates of $Y_{1}$ are $\left(\frac{q}{4}+\frac{p}{2}+\frac{1}{2}, \frac{q}{4} \sqrt{3}\right)$. Substituting $q=2 k+1$, we obtain $Y_{1}\left(\frac{k+p+1}{2}+\frac{1}{4},\left(\frac{k}{2}+\frac{1}{4}\right) \sqrt{3}\right)$. From the value of the second coordinate we have that $Y_{1}$ belongs to the line that passes in the middle between the horizontal lines $y=k \frac{\sqrt{3}}{2}$ and $y=(k+1) \frac{\sqrt{3}}{2}$. From the value of the first coordinate of $Y_{1}$ it follows that $Y_{1}$ is the center of $A_{1} A_{3}$, or $A_{3} A_{2}$, or $A_{2} A_{4}$, or $A_{4} A_{1}$ (Figure 7 ).

Similarly consider the midpoint $Y_{2}$ of $X_{2} X_{1}^{\prime}$. The coordinates of $Y_{2}$ are $\left(\frac{3 q}{4}+\frac{3 p}{2}+\frac{1}{2}, \frac{3 q}{4} \sqrt{3}\right)$. Then $Y_{2}$ is the midpoint of the edge that is opposite to the edge with $Y_{1}$.

Now we shall prove that for any closed geodesic $\gamma$ in the regular tetrahedron there exists the closed geodesic $\gamma_{0}$ equivalent to $\gamma$ and passing through the midpoint of the edge.

A geodesic equivalent to $\gamma$ is characterized by the equation $y=\frac{q \sqrt{3}}{q+2 p}(x-\mu)$, where $p, q$ are fixed coprime integers and there exist $\mu_{1}, \mu_{2} \in[0,1]$ such that $\mu_{1}<\mu<\mu_{2}$. Notice that the lines

$$
\begin{aligned}
& \gamma_{1}: y=\frac{q \sqrt{3}}{q+2 p}\left(x-\mu_{1}\right), \\
& \gamma_{2}: y=\frac{q \sqrt{3}}{q+2 p}\left(x-\mu_{2}\right)
\end{aligned}
$$

pass through the vertices of the tiling. It follows that there exist the integer numbers $c_{1}$ and $c_{2}$ such that the points $P_{1}\left(c_{1} \frac{q+2 p}{2 q}+\mu_{1}, c_{1} \frac{\sqrt{3}}{2}\right)$ and $P_{2}\left(c_{2} \frac{q+2 p}{2 q}+\mu_{2}, c_{2} \frac{\sqrt{3}}{2}\right)$ are the vertices of the tilling and $\gamma_{1}$ passes through $P_{1}$ and $\gamma_{2}$ passes through $P_{2}$.

Consider the closed geodesic $\gamma_{0}$ equivalent to $\gamma$ such that the equation of $\gamma_{0}$ is

$$
y=\frac{q \sqrt{3}}{q+2 p}\left(x-\frac{\mu_{1}+\mu_{2}}{2}\right) .
$$

It is easy to proof that the point $P_{0}$ with coordinates $\left(\frac{c_{1}+c_{2}}{2} \frac{q+2 p}{2 q}+\frac{\mu_{1}+\mu_{2}}{2}, \frac{c_{1}+c_{2}}{2} \frac{\sqrt{3}}{2}\right)$ belongs to $\gamma_{0}$. Let us show that $P_{0}$ is a midpoint of some edge of the tiling. Consider three cases.

1) Both of the points $P_{1}$ and $P_{2}$ belong to the line $A_{1} A_{2}$.

It follows that $c_{1}=2 k_{1}, c_{2}=2 k_{2}$ and $x_{1}=l_{1}, x_{2}=l_{2}$, where $k_{1}, k_{2}, l_{1}, l_{2}$ are integers, and $P_{0}$ has 
the coordinates $\left(\frac{l_{1}+l_{2}}{2},\left(k_{1}+k_{2}\right) \frac{\sqrt{3}}{2}\right)$. Since $\gamma_{0}$ doesn't pass through the vertices, we are reduced to two cases. If $k_{1}+k_{2}=2 k_{0}$, then $l_{1}+l_{2}=2 l_{0}-1$, and the coordinates of $P_{0}$ are $\left(l_{0}-\frac{1}{2}, k_{0} \sqrt{3}\right)$. If $k_{1}+k_{2}=2 k_{0}-1$, then $l_{1}+l_{2}=2 l_{0}$, and $P_{0}$ has the coordinates $\left(l_{0},\left(2 k_{0}-1\right) \frac{\sqrt{3}}{2}\right)$. In both cases we get that $P_{0}$ is the midpoint of some edge.

2) Both of the points $P_{1}, P_{2}$ belong to the line $A_{3} A_{4}$.

It follows that $c_{1}=2 k_{1}+1, c_{2}=2 k_{2}+1$ and $x_{1}=l_{1}+\frac{1}{2}, x_{2}=l_{2}+\frac{1}{2}$, where $k_{1}, k_{2}, l_{1}, l_{2}$ are integers, and the coordinates of $P_{0}$ are $\left(\frac{l_{1}+l_{2}+1}{2},\left(k_{1}+k_{2}+1\right) \frac{\sqrt{3}}{2}\right)$. As above, since $\gamma_{0}$ doesn't pass through the vertices, we get two cases. If $k_{1}+k_{2}+1=2 k_{0}-1$, then $l_{1}+l_{2}+1=2 l_{0}$, and $P_{0}$ has the coordinates $\left(l_{0},\left(2 k_{0}-1\right) \frac{\sqrt{3}}{2}\right)$. If $k_{1}+k_{2}+1=2 k_{0}$, then $l_{1}+l_{2}+1=2 l_{0}-1$ and the coordinates of $P_{0}$ are $\left(l_{0}-\frac{1}{2}, k_{0} \sqrt{3}\right)$. In all this cases the point $P_{0}$ is the midpoint of some edge.

3) The point $P_{1}$ belongs to the line $A_{1} A_{2}$ and the point $P_{2}$ belongs to the line $A_{3} A_{4}$. It follows that $c_{1}=2 k_{1}, c_{2}=2 k_{2}+1$, and $x_{1}=l_{1}, x_{2}=l_{2}+\frac{1}{2}$, where $k_{1}, k_{2}, l_{1}, l_{2}$ are integers. The coordinates of $P_{0}$ are $\left(\frac{l_{1}+l_{2}}{2}+\frac{1}{4},\left(k_{1}+k_{2}+\frac{1}{2}\right) \frac{\sqrt{3}}{2}\right)$. We get that $P_{0}$ is also the center of some edge.

Finally, we found the closed geodesic $\gamma_{0}$ equivalent to $\gamma$ such that $\gamma_{0}$ passes through the midpoint of some edge. As we proved before, from this it follows that $\gamma_{0}$ passes through the midpoints of two pairs of the opposite edges on the tetrahedron. This completes the proof.

Corollary 4.1. The midpoints of two pairs of the opposite edges partition the geodesic $\gamma_{0}$ into four equals parts.

Corollary 4.2. The development of a regular tetrahedron along a closed geodesic consists of four equal polygons. Any two adjacent polygons coincide modulo the point reflection with respect to the midpoint of their common edge.

Proof. For any closed geodesic $\gamma$ we get the equivalent closed geodesic $\gamma_{0}$ that passes through the midpoints of two pairs of the opposite edges on the tetrahedron. Let the points $X_{1}, X_{2}$ and $Y_{1}, Y_{2}$ be respectively the midpoints of the edges $A_{1} A_{2}, A_{4} A_{3}$ and $A_{2} A_{3}, A_{1} A_{4}$. Assume $\gamma_{0}$ passes through these points (Figure 8).

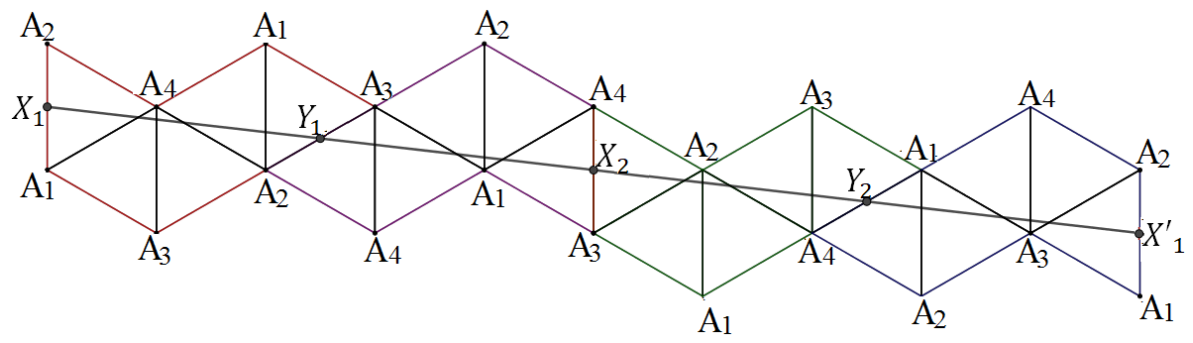

Figure 8

Consider the rotation of the regular tetrahedron by the angle $\pi$ around the line passing through the points $X_{1}$ and $X_{2}$. This rotation is the isometry of the regular tetrahedron. The point $Y_{1}$ is mapped to the point $Y_{2}$. Furthermore the segment of $\gamma_{0}$ that starts at $X_{1}$ on the face $A_{1} A_{2} A_{4}$ is mapped to the segment of $\gamma_{0}$ that starts from the point $X_{1}$ on $A_{1} A_{2} A_{3}$. It follows that the segment $X_{1} Y_{1}$ of the geodesic is mapped to the segment $X_{1} Y_{2}$. For the same reason after the rotation the segments $X_{2} Y_{1}$ and $X_{2} Y_{2}$ of $\gamma_{0}$ swap.

From this rotation we get that the development of the tetrahedron along the segment $Y_{1} X_{1} Y_{2}$ of the geodesic is central-symmetric with respect to the point $X_{1}$. And the development of the tetrahedron through the segment $Y_{1} X_{2} Y_{2}$ is central-symmetric with respect to $X_{2}$. 
Now consider the rotation of the regular tetrahedron by the angle $\pi$ around the line passing through the point $Y_{1}$ and $Y_{2}$. For the same reason we obtain that the development of the tetrahedron along the segment $X_{1} Y_{1} X_{2}$ of geodesic is central-symmetric with respect to $Y_{1}$, and the development along the segment $X_{1} Y_{2} X_{2}$ is central-symmetric with respect to $Y_{2}$.

\section{$5 \quad$ Properties of a closed geodesics on regular tetrahedra in Lobachevsky space}

Let us introduce some notaions following [18]. A broken line on a tetrahedron is a curve that consists of the line segments connecting points on the edges of this tetrahedron in consecutine order. A broken line on a tetrahedron is called a generalized geodesic if it is closed and

(1) has not self-intersection points,

(2) passes through more than three edges on the tetrahedron and doesn't pass through its vertices,

(3) segments incident to the same point on the edge belong to the adjacent faces of the tetrahedron.

Lemma 5.1. (V. Yu. Protasov [18]) For every generalized geodesic on a tetrahedron in Euclidean space there exists a simple closed geodesic on a regular tetrahedron in Euclidean space that is equivalent to this generalized geodesic.

We obtain the analog of Lemma 4.1 for hyperbolic space.

Lemma 5.2. A simple closed geodesic on a regular tetrahedron in Lobachevsky space passes through the midpoints of two pairs of the opposite edges on the tetrahedron.

Proof. Assume $\gamma$ is a simple closed geodesic on a regular tetrahedron $A_{1} A_{2} A_{3} A_{4}$ in Lobachevsky space. In the Cayley-Klein model of this space the regular tetrahedron is represented by a regular tetrahedron in Euclidean space as described in Section 2. Then the geodesic $\gamma$ is a generalized geodesic on the regular tetrahedron in Euclidean space. From Lemma 5.1 we get that this generalized geodesic is equivalent to a closed geodesic $\tilde{\gamma}$ on the regular tetrahedron in Euclidean space. From Lemma 4.1 we assume that $\tilde{\gamma}$ passes through the midpoints of two pairs of the opposite edges on this tetrahedron.

Suppose $\tilde{\gamma}$ passes through the midpoints $\tilde{X}_{1}, \tilde{X}_{2}$ of the edges $A_{1} A_{2}$ and $A_{3} A_{4}$. Let $X_{1}, X_{2}$ be the corresponding points on $\gamma$. Consider the development $T$ of the regular tetrahedron in hyperbolic space along $\gamma$ from the point $X_{1}$. Then $\gamma$ is a line segment $X_{1} X_{1}^{1}$ on the development.

Likewise consider the development of the regular tetrahedron in Euclidean space along $\tilde{\gamma}$ from $\tilde{X}_{1}$. From Corollary 4.2 it follows that this development is central-symmetric with respect to the point $\tilde{X}_{2}$.

On the regular tetrahedron in Lobachevsky space denote by $M_{1}, M_{2}$ respectively the midpoints of the edges $A_{1} A_{2}$ and $A_{3} A_{4}$. Consider the rotation of the tetrahedron by the angle $\pi$ around the line passing through the points $M_{1}$ and $M_{2}$. Since this rotation is the isometry of the tetrahedron then the development of this tetrahedron is central symmetric with respect to the point $M_{2}$. Denote by $T_{1}$ and $T_{2}$ respectively the parts of the development along segments $X_{1} X_{2}$ and $X_{2} X_{1}^{1}$.

Consider the rotation by the angle $\pi$ around the point $M_{2}$. Then the part $T_{1}$ coincides with $T_{2}$. The edge $A_{1} A_{2}$ containing the point $X_{1}^{1}$ is mapped to the edge $A_{2} A_{1}$ with the point $X_{1}$. It follows that the point $X_{1}^{1}$ belongs to the edge $A_{1} A_{2}$ of the $T_{1}$, and the lengths of $A_{2} X_{1}$ and $X_{1}^{1} A_{1}$ are equal. 
The edge $A_{3} A_{4}$ rotates to itself but with the opposite orientation. It follows that the point $X_{2}$ of the part $T_{2}$ is mapped to the point $X_{2}^{1}$ of $T_{1}$ such that the lengths of $A_{4} X_{2}$ and $X_{2}^{1} A_{3}$ are equal. Moreover, $\angle X_{1} X_{2} A_{4}=\angle X_{1}^{1} X_{2}^{1} A_{4}$. Since the geodesic is closed, then $\angle A_{1} X_{1} X_{2}=\angle A_{1} X_{1}^{1} X_{2}^{1}$ (Figure 9).

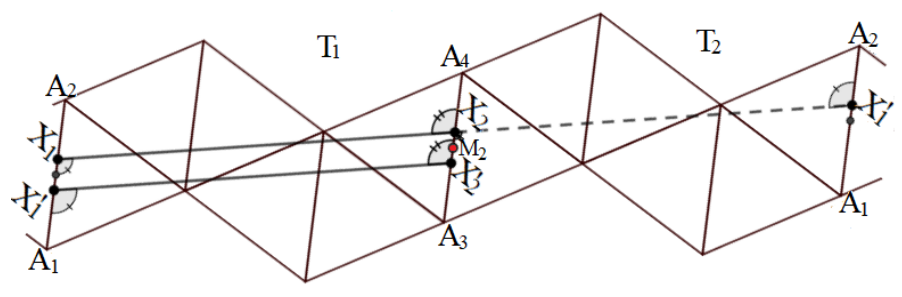

Figure 9

We obtain the quadrilateral $X_{1} X_{2} X_{2}^{1} X_{1}^{1}$ on $T_{1}$ and the sum of its interior angles is equal to $2 \pi$. From Gauss-Bonnet theorem we get that the integral of the Gaussian curvature of Lobachevsky plane over the interior of $X_{1} X_{2} X_{2}^{1} X_{1}^{1}$ is equal to zero. It follows that after the rotation the part $X_{2}^{1} X_{1}^{1}$ of the geodesic coincides with $X_{1} X_{2}$. Hence the points $X_{1}$ and $X_{2}$ are the midpoints of the edges (Figure 9).

In the same way we can proof that $\gamma$ passes through the midpoints of another two opposite edges. This completes the proof.

If $\tilde{\gamma}$ is a closed geodesic on a regular tetrahedron in Euclidean space, then there exist infinetly many closed geodesics equivalent to $\tilde{\gamma}[16]$. It is not true in Lobachevsky space.

Lemma 5.3. If two closed geodesic on the regular tetrahedron in Lobachevsky space are equivalent, then they coincide.

Proof. Let $\gamma_{1}$ be a closed geodesic on a regular tetrhedron in Lobachevsky space. Consider the development of this tetrhedron along $\gamma_{1}$ from a point $X$ of the edge $A_{1} A_{2}$. Then $\gamma_{1}$ corresponds to the segment $X X^{\prime}$ on the development and $\angle X X^{\prime} A_{1}=\angle X^{\prime} X A_{2}$ (Figure 10).

Suppose $\gamma_{2}$ is a closed geodesic equivalent to $\gamma_{1}$ and $\gamma_{2}$ does not coinside with $\gamma_{1}$. Then $\gamma_{2}$ starts at the point $Y$ on the edge $A_{1} A_{2}$ and $Y$ differs from $X$. Since $\gamma_{2}$ and $\gamma_{1}$ pass through the same edges in the same order, then $\gamma_{2}$ corresponds to the segment $Y Y^{\prime}$ on the same development and $\angle Y Y^{\prime} A_{1}=\angle Y^{\prime} Y A_{2}$.

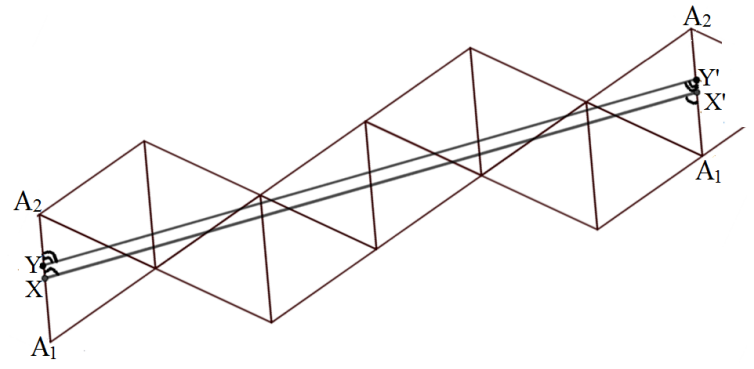

Figure 10

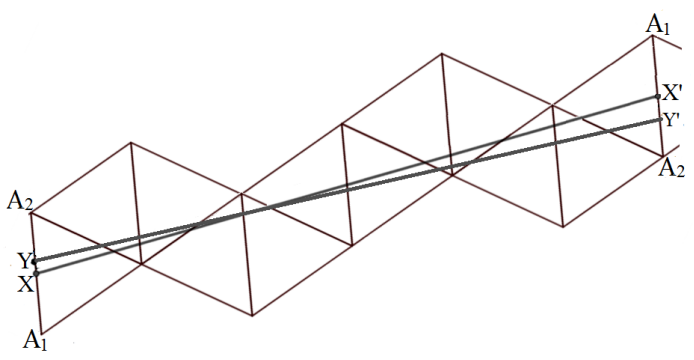

Figure 11

Assume that $Y Y^{\prime}$ intersects $X X^{\prime}$ on the development. It follows that the edge with the points $X$ and $Y$ and the edge with the points $X^{\prime}$ and $Y^{\prime}$ have the opposite orientation. Since the edges with this points correspond to the edge $A_{1} A_{2}$ on the tetrahedron, we obtain that the tetrahedron contains a Mobius strip (Figure 11). It contradicts the orientation of the tetrahedron. 
Thus the segments $Y Y^{\prime}$ and $X X^{\prime}$ do not intersect. We get a quadrilateral $X X^{\prime} Y Y^{\prime}$ (Figure 10. The sum of its interior angles is equal to $2 \pi$. As before from Gauss-Bonnet theorem it follows that $X X^{\prime}$ and $Y Y^{\prime}$ are coincident. It follows that the closed geodesic $\gamma_{2}$ coincides with the closed geodesic $\gamma_{1}$.

\section{Proof of Theorem 1}

Lemma 6.1. (V. Yu. Protasov [18])A simple closed geodesic $\gamma$ on a regular tetrahedron in Euclidean space is uniquely characterized by the two coprime integers $(p, q)$ such that there are $p$ points of $\gamma$ on each of two opposite edges of the tetrahedron, $q$ points of $\gamma$ on each of another two opposite edges, and there are $(p+q)$ points of $\gamma$ on each edges of the third pair of opposite edges.

Remark 2. By two coprime integers $(p, q)$ we mean two integer numbers $p$, $q$ such that $0<$ $p<q$ and $G C D(p, q)=1$, or $p=0, q=1$.

This pair of coprime integers $(p, q)$ is called a type of a simple closed geodesic. Moreover, for any two coprime integers $(p, q)$ there exist infinitely many simple closed geodesics of type $(p, q)$ on a regular tetrahedron in Euclidean space and all of these geodesics are equivalent to each other. From Lemma 4.1 it follows that in the set of geodesics of type $(p, q)$ there exists a geodesic $\tilde{\gamma}$ passing through the midpoints of two pairs of the opposite edges on the tetrahedron. Denote by $\tilde{X}_{1}$ and $\tilde{X}_{2}$ the midpoints of the edges $A_{1} A_{2}$ and $A_{3} A_{4}$ and by $\tilde{Y}_{1}, \tilde{Y}_{2}$ the midpoints of $A_{1} A_{3}$ and $A_{2} A_{4}$. Assume that $\tilde{\gamma}$ passes through $\tilde{X}_{1}, \tilde{X}_{2}$ and $\tilde{Y}_{1}, \tilde{Y}_{2}$. Consider the development of the tetrahedron along $\tilde{\gamma}$ from the point $\tilde{X}_{1}$ through the point $\tilde{Y}_{1}$ to the point $\tilde{X}_{2}$. It is the polygon $\tilde{T}_{1}$ on Euclidean plane. Furthermore, $\tilde{T}_{1}$ is a central-symmetric with respect to $\tilde{Y}_{1}$. The interior angles of $\tilde{T}_{1}$ are equal to $\frac{\pi}{3}$, or $\frac{2 \pi}{3}$, or $\pi$, or $\frac{4 \pi}{3}$. Note that the angle is equal to $\frac{4 \pi}{3}$ if and only if $\tilde{\gamma}$ intersects successively three edges sharing a common vertex.

Now consider the set of congruent regular triangles with the plane angle equal to $\alpha$ on Lobachevsky plane. Put these triangles in the same order as we develop the faces of the regular tetrahedron in Euclidean space along the part $\tilde{X}_{1} \tilde{Y}_{1} \tilde{X}_{2}$ of $\tilde{\gamma}$. In other words, we build a polygon $T_{1}$ on Lobachevsky plane that consists of the triangles ordered in the same way as $\tilde{T}_{1}$ on Euclidean plane. Label the vertices of $T_{1}$ according to the vertices of $\tilde{T}_{1}$. Then the polygon $T_{1}$ corresponds to some development of a regular tetrahedron with the plane angles of the faces equal to $\alpha$ in Lobachevsky space. Moreover, $T_{1}$ is also central-symmetric with respect to the midpoint of the same edge $A_{1} A_{3}$ as the polygon $\tilde{T}_{1}$. By construction, the interior angles at the vertices of $T_{1}$ are equal to $\alpha$ or $2 \alpha$, or $3 \alpha$, or $4 \alpha$.

Consider the midpoints $X_{1}$ and $X_{2}$ of the edges $A_{1} A_{2}$ and $A_{3} A_{4}$ on $T_{1}$ that $X_{1}$ and $X_{2}$ correspond to the points $\tilde{X}_{1}$ and $\tilde{X}_{2}$ on $\tilde{T}_{1}$. Construct the line segment $X_{1} X_{2}$.

Assume $\alpha \in\left(0, \frac{\pi}{4}\right]$. Hence the polygon $T_{1}$ is convex. It follows that the segment $X_{1} X_{2}$ belongs to the interior of the polygon $T_{1}$. Moreover, $X_{1} X_{2}$ passes through the center of symmetry of $T_{1}$.

In the same way consider the development $\tilde{T}_{2}$ of the tetrahedron along the second part $\tilde{X}_{2} \tilde{Y}_{2} \tilde{X}_{1}^{\prime}$ of the geodesic $\tilde{\gamma}$ in Euclidean space. Note that $\tilde{T}_{1}$ and $\tilde{T}_{2}$ are equal polygons (without vertex labeling). Then consider the second copy of $T_{1}$ on hyperbolic plane, denote it by $T_{2}$ and label vertices of $T_{2}$ according to the vertices on $\tilde{T}_{2}$. Mark by $X_{2}$ and $X_{1}^{\prime}$ the midpoints of $A_{3} A_{4}$ and $A_{1} A_{2}$ on $T_{2}$ so that $X_{2}$ and $X_{1}^{\prime}$ correspond to the points $\tilde{X}_{2}$ and $\tilde{X}_{1}^{\prime}$. Since $\alpha \in\left(0, \frac{\pi}{4}\right]$ then the segment $X_{2} X_{1}^{\prime}$ also belongs to the interior of $T$ and passes through its center of symmetry.

These two polygons $T_{1}$ and $T_{2}$ correspond to the development of a regular tetrahedron with the plane angles of the faces equal to $\alpha$ in Lobachevsky space. Then $X_{1} X_{2}$ and $X_{2} X_{1}^{\prime}$ are the geodesic segments on this tetrahedron. Furthermore, on the tetrahedron the point $X_{2}$ of $X_{1} X_{2}$ 
coincide with $X_{2}$ of $X_{2} X_{1}^{\prime}$ and the point $X_{1}$ of the segment $X_{1} X_{2}$ coincide with $X_{1}^{\prime}$ of $X_{2} X_{1}^{\prime}$. Since $T_{1}$ and $T_{2}$ is central symmetric then $\angle X_{1} X_{2} A_{3}=\angle X_{1}^{\prime} X_{2} A_{4}$ and $\angle X_{2} X_{1} A_{1}=\angle X_{2} X_{1}^{\prime} A_{2}$. The segments $X_{1} X_{2}$ and $X_{2} X_{1}^{\prime}$ are equivalent to the geodesic segments $\tilde{X}_{1} \tilde{X}_{2}$ and $\tilde{X}_{2} \tilde{X}_{1}^{\prime}$ on the regular tetrahedron in Euclidian space respectively. Therefore, $X_{1} X_{2}$ and $X_{2} X_{1}^{\prime}$ form a simple closed geodesic $\gamma$ on the regular tetrahedron with the plane angles equal to $\alpha \in\left(0, \frac{\pi}{4}\right]$ in Lobachevsky space.

Increase the angle $\alpha$ from the $\frac{\pi}{4}$. Then the polygon $T$ is not convex because it contains the interior angles equal to $4 \alpha>\pi$.

Let $\alpha_{0}$ be the supremum of the set of $\alpha$ such that the segment $X_{1} X_{2}$ belongs to the interior of $T_{1}$. Assume $\alpha_{0}<\frac{\pi}{3}$. For all $\alpha<\alpha_{0}$ the segment $X_{1} X_{2}$ is a part of a simple closed geodesic $\gamma$ on the regular tetrahedron in Lobachevsky space. The distance from the set of vertices of the tetrahedron to $\gamma$ satisfies inequality (3.9). It follows that there exists sufficiently small $\varepsilon$ such that for $\alpha_{1}=\alpha_{0}+\varepsilon$ the segment $X_{1} X_{2}$ belongs to the interior of $T$. It contradicts to the supremum of this set. Then $\alpha_{0}=\frac{\pi}{3}$.

Finally, we obtain that for all $\alpha \in\left(0, \frac{\pi}{3}\right)$ the segments $X_{1} X_{2}$ and $X_{2} X_{1}^{\prime}$ belong to the interior of $T$. From this it follows

Theorem 1. On a regular tetrahedron in Lobachevsky space for any coprime integers $(p, q)$, $0 \leq p<q$, there exists unique, up to the rigid motion of the tetrahedron, simple closed geodesic of type $(p, q)$. The geodesics of type $(p, q)$ exhaust all simple closed geodesics on a regular tetrahedron in Lobachevsky space.

From Lemma 5.3 it follows the uniqueness of a simple closed geodesic of type $(p, q)$ on a regular tetrahedron in Lobachevsky space. Such geodesic has $p$ points on each of two opposite edges of the tetrahedron, $q$ points on each of another two opposite edges, and $(p+q)$ points on each edges of the third pair of opposite edges. Hence for any coprime integers $(p, q), 0 \leq p<q$, there exist three simple closed geodesic of type $(p, q)$ on a regular tetrahedron in Lobachevsky space. They coincide by the rotation of the tetrahedron by the angle $\frac{2 \pi}{3}$ or $\frac{4 \pi}{3}$ about the altitude constructed from a vertex to the opposite face.

Since any simple closed geodesic on a regular tetrahedron in Lobachevsky space is equivalent to a simple closed geodesic on a regular tetrahedron in Euclidean space, then there are not another simple closed geodesic on a regular tetrahedron in Lobachevsky space.
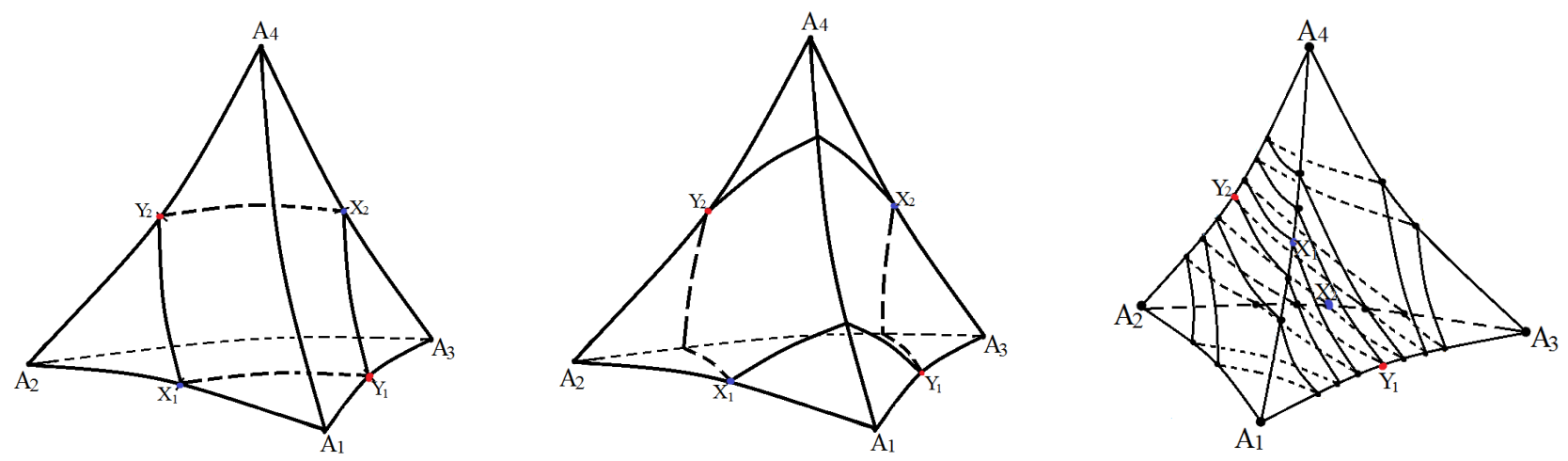

Figure 12. A simple closed geodesics of type $(0,1),(1,1)$ and $(5,2)$ respectively on a regular tetrahedron in Lobachevsky space. 


\section{Proof of Theorem 2}

By vertex of a geodesic we understand a point of this geodesic that belongs to an edge of a tetrahedron. Consider vertices $B_{0}, B_{1}^{1}, B_{1}^{2}$ of the geodesic such that the segments $B_{0} B_{1}^{1}$ and $B_{0} B_{1}^{2}$ are adjacent segments of the geodesic. The vertex $B_{0}$ is called a catching point if $B_{0}, B_{1}^{1}$ and $B_{1}^{2}$ lie on the three edges sharing a common vertex on the tetrahedron and $B_{0}, B_{1}^{1}, B_{1}^{2}$ are the nearest vertices of geodesic to this vertex of the tetrahedron.

In [18] V. Yu. Protasov shows following property of a simple closed geodesic on a regular tetrahedron in Euclidean space.

Lemma 7.1. [18] Let $\gamma_{1}^{1}, \gamma_{1}^{2}$ be the segments of a simple closed geodesic $\gamma$ comming out from a catching point on a regular tetrahedron in Euclidean space. The segments $\gamma_{2}^{2}, \gamma_{2}^{2}$ of $\gamma$ are the segments follow $\gamma_{1}^{1}, \gamma_{1}^{2}$ respectively and so on. Then for each $k=2, \ldots, 2 p+2 q-1$ the segments $\gamma_{k}^{1}, \gamma_{k}^{2}$ belong to the same face of the tetahedron and there are no points of $\gamma$ between $\gamma_{k}^{1}$ and $\gamma_{k}^{2}$. The last segments $\gamma_{2 p+2 q}^{1}, \gamma_{2 p+2 q}^{2}$ share the second catching point.

Since any simple closed geodesic $\gamma$ on a regular tetrahedron in Lobachevsky space is equivalent to a simple closed geodesic on a regular tetrahedron in Euclidean space, then $\gamma$ also satisfy Lemma 7.1 .

Lemma 7.2. If the length of a simple closed geodesic of type $(p, q)$ on a regular tetrahedron in Lobachevsky space is not greater than $L$, then

$$
p+q \leq \frac{3}{4} \frac{L-2 \ln \left(\frac{2 \pi^{3}-(\pi-3 \alpha)^{3}\left(1-\frac{4 \alpha^{2}}{\pi^{2}}\right)}{2 \pi^{3}-(\pi-3 \alpha)^{3}\left(1+\frac{4 \alpha^{2}}{\pi^{2}}\right)}\right)}{\ln \left(\frac{2 \pi^{3}-(\pi-3 \alpha)^{3}\left(1-\frac{\alpha^{2}}{\pi^{2}}\right)}{2 \pi^{3}-(\pi-3 \alpha)^{3}\left(1+\frac{\alpha^{2}}{\pi^{2}}\right)}\right)+\ln \left(\frac{3 \pi-3 \alpha}{\pi+3 \alpha}\right)}+2
$$

where $\alpha$ is the plane angle of a face of the tetrahedron.

Proof. 1. Consider a regular tetrahedron $A_{1} A_{2} A_{3} A_{4}$ with the plane angle of a face equal to $\alpha$ in Lobachevsky space. Let $\gamma$ be a simple closed geodesic of type $(p, q)$ on this tetrahedron. Assume that the vertices $B_{1}, B_{2}, B_{3}$ of $\gamma$ belong to the edges $A_{4} A_{1}, A_{4} A_{2}, A_{4} A_{3}$ respectively and these vertices are the nearest to the vertex $A_{4}$. Thus the segments $B_{2} B_{1}$ and $B_{2} B_{3}$ corespond to the segments $\gamma_{1}^{1}$ and $\gamma_{1}^{2}$ sharing a catching point $B_{2}$.

Consider the development of the faces $A_{1} A_{4} A_{2}$ and $A_{2} A_{4} A_{3}$ on hyperbolic plane. The segments $\gamma_{1}^{1}$ and $\gamma_{1}^{2}$ correspond to the one segment $B_{1} B_{3}$ on this development. Without loss of generality we assume that the length of $A_{4} B_{1}$ is not less than the length of $A_{4} B_{3}$.

Let us check that the function of the distance between $A_{4}$ and points on $\gamma$ attains its minimum at the point $H$ belonging to the segment $B_{1} B_{3}$. Suppose it is not true. We will extend the geodesic $\gamma$ from the point $B_{3}$ on the face $A_{1} A_{4} A_{3}$. From Corollary 3.1 we obtain that the distance between $A_{4}$ and $\gamma$ is decrease. Therefore, $\gamma$ intersects the edge $A_{1} A_{4}$ on the point belonging to the segment $A_{4} B_{1}$. But the vertex $B_{1}$ is the nearest to $A_{4}$ vertex of $\gamma$ on the edge $A_{1} A_{4}$. We get a contradiction. Then the point $H$ belonging to the segment $B_{1} B_{3}$ and $A_{4} H$ is perpendicular to $B_{1} B_{3}$.

Denote the angle $\angle B_{2} A_{4} H$ by $\beta$ and $\beta \in[-\alpha, \alpha]$ (Figure 13). Consider the length of $B_{1} B_{3}$ as a fuction of $\beta$. From equation (3.3) applied to the triangles $B_{1} A_{4} H$ and $B_{3} A_{4} H$ we obtain

$$
\begin{aligned}
& \tanh \left|B_{1} H\right|=\sinh \left|A_{4} H\right| \tan (\alpha+\beta) . \\
& \tanh \left|B_{3} H\right|=\sinh \left|A_{4} H\right| \tan (\alpha-\beta) .
\end{aligned}
$$

From equations $(7.2)$ and $(7.3)$ it follows that

$$
\left|B_{1} B_{3}\right|=\operatorname{artanh}\left(\sinh \left|A_{4} H\right| \tan (\alpha+\beta)\right)+\operatorname{artanh}\left(\sinh \left|A_{4} H\right| \tan (\alpha-\beta)\right) .
$$




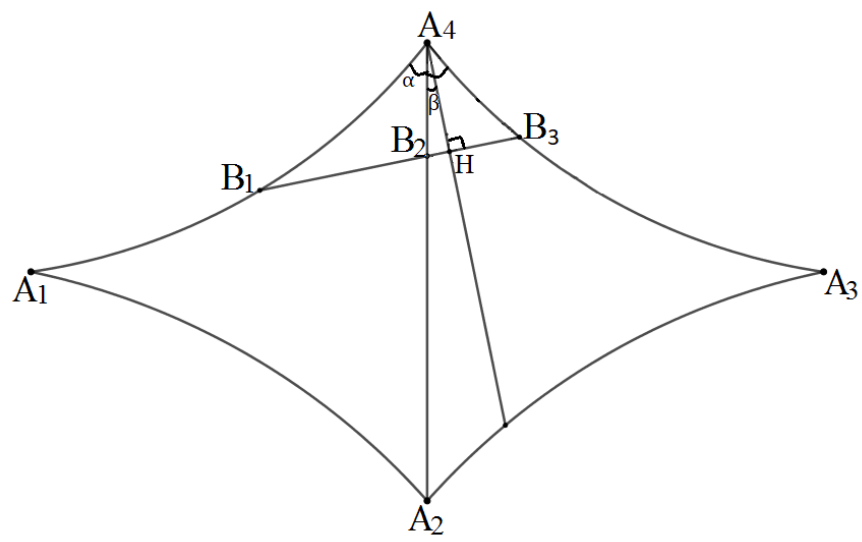

Figure 13

Find the first derivative of the function in the right side of $(7.4)$, set the derivative equal to zero and solve for $\beta$. We obtain that the length of $B_{1} B_{3}$ attains its minimum at $\beta=0$ and this minimum is

$$
\left|B_{1} B_{3}\right|_{\text {min }}=2 \operatorname{artanh}\left(\sinh \left|A_{4} H\right| \tan \alpha\right) .
$$

From Lemma 3.4 we get that the distance between $A_{4}$ and $\gamma$ satisfies follow inequality

$$
\tanh \left|A_{4} H\right|>\cos \frac{3 \alpha}{2} \cos \frac{\alpha}{2} \frac{\sqrt{2 \cos \alpha-1}}{\cos \alpha} .
$$

Using $\sinh \left|A_{4} H\right|=\frac{\tanh \left|A_{4} H\right|}{\sqrt{1-\tanh ^{2}\left|A_{4} H\right|}}$, we obtain

$$
\sinh \left|A_{4} H\right|>\frac{\cos \alpha \cos \frac{3 \alpha}{2} \sqrt{\cos ^{3} \frac{3 \alpha}{2} \cos \frac{\alpha}{2}}}{\cos ^{2} \alpha-\cos ^{3} \frac{3 \alpha}{2} \cos \frac{\alpha}{2}} .
$$

Combining $(7.6)$ and $(7.5)$, we get follow estimation of the length $B_{1} B_{3}$

$$
\tanh \frac{\left|B_{1} B_{3}\right|}{2}>\frac{\sin \alpha \cos \frac{3 \alpha}{2} \sqrt{\cos ^{3} \frac{3 \alpha}{2} \cos \frac{\alpha}{2}}}{\cos ^{2} \alpha-\cos ^{3} \frac{3 \alpha}{2} \cos \frac{\alpha}{2}} .
$$

The length of the segments $\gamma_{2 p+2 q}^{1}$ and $\gamma_{2 p+2 q}^{2}$ comming out from the second catching point also satisfies inequality (7.7).

2. Consider the development of the tetrahedron along the segments $\gamma_{i}^{1}, i=2, \ldots, 2 p+$ $2 q-1$ of the geodesic in hyperbolic space. These segments form a line segment $\gamma^{1}$ inside the development. From Lemma 7.1 we have that the segments $\gamma_{i}^{2}, i=2, \ldots, 2 p+2 q-1$ intersect the same sequence of the edges. It follows that $\gamma_{i}^{2}, i=2, \ldots, 2 p+2 q-1$ form the segment $\gamma^{2}$ inside the development and $\gamma^{2}$ does not intersect $\gamma^{1}$.

The development along $\gamma^{1}$ consist of $(2 q+2 p-2)$ faces of the tetrahedron. Consider three consecutive faces of the development containig the segments $\gamma_{i-1}^{1}, \gamma_{i}^{1}, \gamma_{i+1}^{1}, i=3 \ldots 2 p+2 q-2$. Since $\gamma$ is a simple closed geodesic, it follows that $\gamma^{1}$ does not pass throught four edges sharing a common vertex of the tetrahedron. Therefore, the segments $\gamma_{i-1}^{1}, \gamma_{i}^{1}, \gamma_{i+1}^{1}$ intersect two opposite edges of the tetrahedron. Assume that $\gamma_{i-1}^{1}, \gamma_{i}^{1}$ and $\gamma_{i+1}^{1}$ intersect the edges $A_{4} A_{3}, A_{4} A_{1}, A_{4} A_{2}$ and $A_{2} A_{3}$ at the points $B_{i-2}, B_{i-1}, B_{i}$ and $B_{i+1}$ respectively (Figure 14).

Similarly to the estimation of the length $B_{1} B_{3}$, we obtain following inequality for the length of $B_{i-2} B_{i-1}$.

$$
\tanh \frac{\left|B_{i-2} B_{i-1}\right|}{2}>\frac{\tan \frac{\alpha}{2} \cos \alpha \cos \frac{3 \alpha}{2} \sqrt{\cos ^{3} \frac{3 \alpha}{2} \cos \frac{\alpha}{2}}}{\cos ^{2} \alpha-\cos ^{3} \frac{3 \alpha}{2} \cos \frac{\alpha}{2}} .
$$




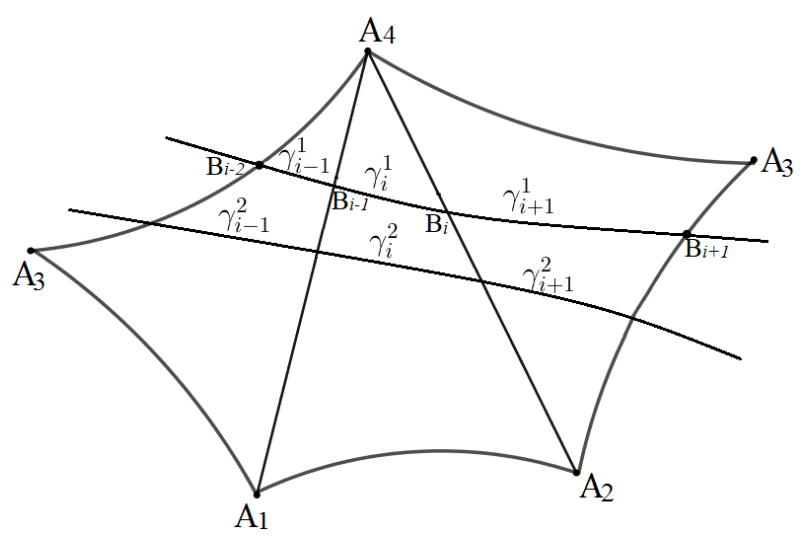

Figure 14

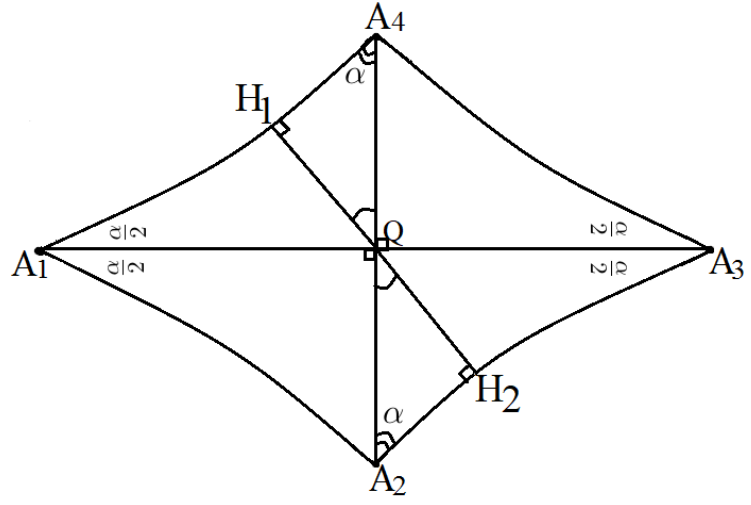

Figure 15

For evaluating the length of the segment $B_{i-1} B_{i+1}$ consider the development of two adjacent faces $A_{4} A_{2} A_{1}$ and $A_{4} A_{2} A_{3}$ containing this segment. Denote by $Q$ the midpoint of the edge $A_{2} A_{4}$. On the edges $A_{4} A_{1}$ and $A_{2} A_{3}$ mark the points $H_{1}$ and $H_{2}$ respectively so that $Q H_{1}$ is perpendicular to $A_{4} A_{1}$ and $Q H_{2}$ is perpendicular to $A_{2} A_{3}$. Since $\angle H_{1} A_{4} Q=\angle H_{2} A_{2} Q=\alpha<\frac{\pi}{2}$ and the sum of the interior angles of the hyperbolic triangle is less then $\pi$, it follows that the segments $Q H_{1}, Q H_{2}$ belong to the triangles $A_{1} Q A_{4}, A_{3} Q A_{2}$ respectively.

The segment $A_{1} A_{3}$ is perpendicular to $A_{4} A_{2}$ at the point $Q$ (Figure 15). The lengths of $A_{1} Q$ and $Q A_{3}$ are equal. It follows that the faces $A_{1} A_{2} A_{4}, A_{2} A_{4} A_{3}$ coincide modulo the rotation by the angle $\pi$ about $Q$. After this rotation the point $H_{2}$ on $A_{2} A_{3}$ is mapped to the point $H_{1}$ on $A_{4} A_{1}$. Then $\angle A_{4} Q H_{1}=\angle A_{2} Q H_{2}$. We obtain that $H_{1} Q H_{2}$ is a straight line segment and the function of a distance between $A_{1} A_{4}$ and $A_{2} A_{3}$ attains its minimum at $H_{1} H_{2}$. From equation (3.4) applied to the triangle $Q H_{1} A_{4}$ we obtain

$$
\sinh \left|Q H_{1}\right|=\sinh \left|Q A_{4}\right| \sin \alpha=\sinh \frac{a}{2} \sin \alpha .
$$

From equation (2.4) we get

$$
\sinh \left|Q H_{1}\right|=\frac{\sqrt{2 \cos \alpha-1}}{2 \sin \frac{\alpha}{2}} \sin \alpha=\cos \frac{\alpha}{2} \sqrt{2 \cos \alpha-1} .
$$

Let us check that the distance from the set of the vertices of the tetrahedron to $H_{1} H_{2}$ satisfies necessary condition (3.6). From the hyperbolic Pythagorean theorem (3.1) applied to the triangle $A_{4} H_{1} Q$ we get

$$
\cosh \left|A_{4} H_{1}\right|=\frac{\cosh \left|A_{4} Q\right|}{\cosh \left|Q H_{1}\right|}=\frac{\cosh \frac{a}{2}}{\sqrt{1+\sinh ^{2}\left|Q H_{1}\right|}} .
$$

Using (2.3) and (7.10), we obtain

$$
\cosh \left|A_{4} H_{1}\right|=\frac{1}{2 \sin \frac{\alpha}{2} \sqrt{\cos ^{2} \alpha+\cos ^{2} \frac{\alpha}{2}}} .
$$

Transforming 7.11, we get

$$
\tanh \left|A_{4} H_{1}\right|=1-4 \sin ^{2} \frac{\alpha}{2}\left(\cos ^{2} \alpha+\cos ^{2} \frac{\alpha}{2}\right) .
$$

From tanh $\left|A_{4} H_{1}\right|>\cos \frac{3 \alpha}{2} \cos \frac{\alpha}{2} \frac{\sqrt{2 \cos \alpha-1}}{\cos \alpha}$ it follows that the necessary condition 3.6 is satisfied. 
Therefore, from 7.10 we get

$$
\sinh \frac{\left|B_{i-1} B_{i+1}\right|}{2} \geq \cos \frac{\alpha}{2} \sqrt{2 \cos \alpha-1} .
$$

Transforming the last inequality, we obtain

$$
\tanh \frac{\left|B_{i-1} B_{i+1}\right|}{2} \geq \frac{\cos \frac{\alpha}{2} \sqrt{2 \cos \alpha-1}}{\sqrt{\cos ^{2} \alpha+\cos ^{2} \frac{\alpha}{2}}} .
$$

Since the length of a simple closed geodesic $\gamma$ of type $(p, q)$ on a regular tetrahedron in Lobachevsky space is not greater than $L$, we get

$$
L \geq 2\left(\frac{2 p+2 q-4}{3}\right)\left(\left|B_{i-2} B_{i-1}\right|+\left|B_{i-1} B_{i+1}\right|\right)+2\left|B_{1} B_{3}\right| .
$$

Using (7.7), (7.8), (7.13), we obtain

$$
\begin{gathered}
L \geq 8\left(\frac{p+q-2}{3}\right)\left(\operatorname{artanh}\left(\frac{\tan \frac{\alpha}{2} \cos \alpha \cos \frac{3 \alpha}{2} \sqrt{\cos ^{3} \frac{3 \alpha}{2} \cos \frac{\alpha}{2}}}{\cos ^{2} \alpha-\cos ^{3} \frac{3 \alpha}{2} \cos \frac{\alpha}{2}}\right)+\operatorname{artanh}\left(\frac{\cos \frac{\alpha}{2} \sqrt{2 \cos \alpha-1}}{\sqrt{\cos ^{2} \alpha+\cos ^{2} \frac{\alpha}{2}}}\right)\right) \\
+4 \operatorname{artanh}\left(\frac{\sin \alpha \cos \frac{3 \alpha}{2} \sqrt{\cos ^{3} \frac{3 \alpha}{2} \cos \frac{\alpha}{2}}}{\cos ^{2} \alpha-\cos ^{3} \frac{3 \alpha}{2} \cos \frac{\alpha}{2}}\right) .
\end{gathered}
$$

Combining inequalities $3.10,3.12$ and $\cos \frac{\alpha}{2}>\frac{\sqrt{3}}{2}$ for $0<\alpha<\frac{\pi}{3}$ with $(7.7),(7.8$ and (7.13, we obtain

$$
\begin{gathered}
\tanh \frac{\left|B_{1} B_{3}\right|}{2} \geq \frac{2 \alpha}{\pi} \sqrt{\frac{(\pi-3 \alpha)^{3}}{2 \pi^{3}-(\pi-3 \alpha)^{3}}} ; \\
\tanh \frac{\left|B_{i-2} B_{i-1}\right|}{2} \geq \frac{\alpha}{\pi} \sqrt{\frac{(\pi-3 \alpha)^{3}}{2 \pi^{3}-(\pi-3 \alpha)^{3}}} ; \\
\tanh \frac{\left|B_{i-1} B_{i+1}\right|}{2} \geq \frac{\sqrt{\pi-3 \alpha}}{4 \sqrt{\pi}} .
\end{gathered}
$$

Transforming these equations, we obtain

$$
\begin{gathered}
\left|B_{1} B_{3}\right| \geq \ln \left(\frac{2 \pi^{3}-(\pi-3 \alpha)^{3}\left(1-\frac{4 \alpha^{2}}{\pi^{2}}\right)}{2 \pi^{3}-(\pi-3 \alpha)^{3}\left(1+\frac{4 \alpha^{2}}{\pi^{2}}\right)}\right) ; \\
\left|B_{i-2} B_{i-1}\right| \geq \ln \left(\frac{2 \pi^{3}-(\pi-3 \alpha)^{3}\left(1-\frac{\alpha^{2}}{\pi^{2}}\right)}{2 \pi^{3}-(\pi-3 \alpha)^{3}\left(1+\frac{\alpha^{2}}{\pi^{2}}\right)}\right) ; \\
\left|B_{i-1} B_{i+1}\right| \geq \ln \left(\frac{3 \pi-3 \alpha}{\pi+3 \alpha}\right) .
\end{gathered}
$$

Putting (7.15), (7.16), (7.17) in (7.14), we have

$$
L \geq 4\left(\frac{p+q-2}{3}\right)\left(\ln \left(\frac{2 \pi^{3}-(\pi-3 \alpha)^{3}\left(1-\frac{\alpha^{2}}{\pi^{2}}\right)}{2 \pi^{3}-(\pi-3 \alpha)^{3}\left(1+\frac{\alpha^{2}}{\pi^{2}}\right)}\right)+\ln \left(\frac{3 \pi-3 \alpha}{\pi+3 \alpha}\right)\right)+2 \ln \left(\frac{2 \pi^{3}-(\pi-3 \alpha)^{3}\left(1-\frac{4 \alpha^{2}}{\pi^{2}}\right)}{2 \pi^{3}-(\pi-3 \alpha)^{3}\left(1+\frac{4 \alpha^{2}}{\pi^{2}}\right)}\right) \text {. }
$$

From (7.18) it follows required estimation (7.1). This complete the proof. 
Euler's function $\phi(n)$ is equal to the number of positive integers not greater than $n$ and prime to $n \in \mathbb{N}$. From [23, Th. 330.] we know

$$
\sum_{n=1}^{x} \phi(n)=\frac{3}{\pi^{2}} x^{2}+O(x \ln x)
$$

where $O(x \ln x)<C x \ln x$, when $x \rightarrow+\infty$.

Denote by $\psi(x)$ a number of pair of coprime integers $(p, q)$ such that $p<q$ and $p+q \leq x$, $x \in \mathbb{R}$.

Lemma 7.3. The asymptotic behavior of $\psi(x)$ is

$$
\psi(x)=\frac{3}{2 \pi^{2}} x^{2}+O(x \ln x),
$$

where $O(x \ln x)<C x \ln x$ when $x \rightarrow+\infty$.

Proof. Suppose $\hat{\psi}(y)$ is equal to the number of pair of coprime integers $(p, q)$ such that $p<q$ and $p+q=y, y \in \mathbb{N}$. From the definitions we get

$$
\psi(x)=\sum_{y=1}^{x} \hat{\psi}(y)
$$

It is easy to proof that if $(p, q)=1$ and $p+q=y$, then $(p, y)=1$ and $(q, y)=1$. For instance, if $(p, y)=s$, then $p=p_{1} s$ and $y=y_{1} s$. It follows that $q=y-p=\left(y_{1}-q_{1}\right) s$. It contradicts to $(p, q)=1$.

Consider Euler's function $\phi(y)$. It is not hard to prove that if $k<y$ and $k$ is prime to $y$, then $y-k$ is prime to $y$ and $y-k$ is prime to $k$. If $(y-k, y)=s$, then $y-k=r_{1} s$ and $y=r_{2} s$. It follows that $k=\left(r_{2}-r_{1}\right) s$. It contradicts to $(k, y)=1$.

Therefore, we obtain that the set of integers not greater than and prime to $y$ are separated into the pairs of coprime integers $(p, q)$ such that $p<q$ and $p+q=y$ It follows that $\phi(y)$ is even and $\hat{\psi}(y)=\frac{1}{2} \phi(y)$. From 7.21 we have

$$
\psi(x)=\frac{1}{2} \sum_{y=1}^{x} \phi(y) .
$$

Using (7.19), we obtain required formula 7.20 ).

Denote by $N(L, \alpha)$ a number of simple closed geodesics of length not greater than $L$ on a regular tetraedron in Lobachevsky space with the plane angles of the faces equal to $\alpha$. For each pair of coprime integers $(p, q), p<q$ corresponds three simple closed geodesics on a regular tetraedron in hyperbolic space. From Lemma 7.2 we obtain

$$
N(L, \alpha)=3 \psi\left(\frac{3}{4} \frac{L-2 \ln \left(\frac{2 \pi^{3}-(\pi-3 \alpha)^{3}\left(1-\frac{4 \alpha^{2}}{\pi^{2}}\right)}{2 \pi^{3}-(\pi-3 \alpha)^{3}\left(1+\frac{4 \alpha^{2}}{\pi^{2}}\right)}\right)}{\ln \left(\frac{2 \pi^{3}-(\pi-3 \alpha)^{3}\left(1-\frac{\alpha^{2}}{\pi^{2}}\right)}{2 \pi^{3}-(\pi-3 \alpha)^{3}\left(1+\frac{\alpha^{2}}{\pi^{2}}\right)}\right)+\ln \left(\frac{3 \pi-3 \alpha}{\pi+3 \alpha}\right)}+2\right)
$$

Using (7.20), we get

$$
N(L, \alpha)=\frac{27 L^{2}}{32 \pi^{2}\left(\ln \left(\frac{2 \pi^{3}-(\pi-3 \alpha)^{3}\left(1-\frac{\alpha^{2}}{\pi^{2}}\right)}{2 \pi^{3}-(\pi-3 \alpha)^{3}\left(1+\frac{\alpha^{2}}{\pi^{2}}\right)}\right)+\ln \left(\frac{3 \pi-3 \alpha}{\pi+3 \alpha}\right)\right)^{2}}+O(L \ln L), \text { при } L \rightarrow+\infty .
$$

Thus we proved follow theorem. 
Theorem 2. Let $N(L, \alpha)$ be a number of simple closed geodesics of length not greater than $L$ on a regular tetraedron with plane angles of the faces equal to $\alpha$ in Lobachevsky space. Then there exists the function $c(\alpha)$ such that

$$
N(L, \alpha)=c(\alpha) L^{2}+O(L \ln L)
$$

where $O(L \ln L) \leq C L \ln L$ when $L \rightarrow+\infty$,

$$
\begin{gathered}
c(\alpha)=\frac{27}{32 \pi^{2}} \frac{1}{\left(\ln \left(\frac{2 \pi^{3}-(\pi-3 \alpha)^{3}\left(1-\frac{\alpha^{2}}{\pi^{2}}\right)}{2 \pi^{3}-(\pi-3 \alpha)^{3}\left(1+\frac{\alpha^{2}}{\pi^{2}}\right)}\right)+\ln \left(\frac{3 \pi-3 \alpha}{\pi+3 \alpha}\right)\right)^{2}}, \\
\lim _{\alpha \rightarrow \frac{\pi}{3}} c(\alpha)=+\infty ; \quad \lim _{\alpha \rightarrow 0} c(\alpha)=\frac{27}{32(\ln 3)^{2} \pi^{2}} .
\end{gathered}
$$

If $\alpha$ goes to zero, then the vertices of the tetrahedron tends to infinity. The limiting surface is non-compact surface with complete regular Riemannian metric of constant negative curvature. Note that the limiting surface is homeomorphic to a sphere with four points at infinity. Then the genus of imiting surface is equal to zero. I. Rivin show that on such surface the number of simple closed geodesics of length no greater than $L$ is of order $L^{2}$.

From the equations 7.23 and 7.22 we obtain that if $\alpha$ goes to zero, then the number $N(L, \alpha)$ is asymptotic to $L^{2}$ when $L \rightarrow+\infty$. Hence, our results are consistent with the results of I. Rivin.

We are grateful to V. A. Gorkavyy for valuable discussions.

\section{References}

[1] G. D. Birkhoff, Dynamical Systems, American mathematical society, Volume IX, 1927.

[2] L. A. Lyusternik and L. G. Shnirel'man, "Sur le probleme de troix geodesique fermees sur les surfaces de genre 0", C. R. Acad. Sci. Paris, 189 (1929), 269-271.

[3] L. A. Lyusternik, L. G. Shnirel'man, "Topological methods in variational problems and their application to the differential geometry of surfaces", Uspekhi Mat. Nauk, 2:1(17) (1947), 166-217

[4] I. A. Taimanov, "On the existence of three nonselfintersecting geodesics on manifolds homeomorphic to the 2-sphere", Russian Academy of Sciences. Izvestiya Mathematics, 40:3 (1993), 565-590.

[5] A. I. Fet and L. A. Lusternik, "Variational problems on closed manifolds" Dokl. Akad. Nauk SSSR, 8:1 (1951), 17-20.

[6] A. I. Fet, "A periodic problem in the calculus of variations", Dokl. Akad. Nauk SSSR (N.S.) 160 (1965), 287-289 ; Soviet Mathematics 6, 85-88 (1965).

[7] W. Klingenberg, Lectures on Closed Geodesics Springer-Verlag Berlin Heidelberg, 1978.

[8] Heinz Huber, "Zur analytischen Theorie hyperbolischen Raumformen und Bewegungsgruppen" Mathematische Annalen, 138:1 (1959), 1-26.

[9] Heinz Huber, "Zur analytischen Theorie hyperbolischen Raumformen und Bewegungsgruppen II" Mathematische Annalen, 143, 1961, 463-464. 
[10] Ya. G. Sinai, "Asymptotic behavior of closed geodesics on compact manifolds with negative curvature" Am. Math. Soc., Transl. II , 73 (1968), 227-250.

[11] G. A. Margulis, "Applications of ergodic theory to the investigation of manifolds of negative curvature" Functional Analysis and Its Applications, 3:4 (1969), 335-336.

[12] I. Rivin, "Simple curves on surfaces" Geometriae Dedicata , 87:1-3 (2001), 345-360.

[13] M. Mirzakhani, "Growth of the number of simple closed geodesics on hyperbolic surfaces" Annals of Mathematics, 168 (2008), 97-125.

[14] A. Cotton, D. Freeman, A. Gnepp, T. Ng, J. Spivack, C. Yoder "The isoperimetric problem on some singular surfaces" J. Aust. Math. Soc., 78:2 (2005), 167-197.

[15] K. Lawson, J. Parish , C. Traub, A. Weyhaupt "Coloring graphs to classify simple closed geodesics on convex deltahedra" International Journal of Pure and Apllied Mathematics, 89 (2013), 1-19.

[16] D. B. Fuchs, E. Fuchs, "Closed geodesics on regular polyhedra" Mosc. Math. J., 7:2 (2007), 265-279.

[17] D. B. Fuchs, "Geodesics on a regular dodecahedron" Preprints of Max Planck Institute for Mathematics, Bonn, 91 (2009), 1-14.

[18] V. Yu. Protasov, "Closed geodesics on the surface of a simplex" Sbornik: Mathematics, 198:2 (2007), 243-260.

[19] A. D. Alexandrov, Convex Polyhedra, Springer, 2005.

[20] Yu. D. Burago, V. A. Zalgaller, Introduction to Riemannian geometry, L, Nauka, 1994.

[21] J. Cannon, W. Floyd, R. Kenyon, W. Parry Hyperbolic Geometry, Flavors of Geometry, MSRI Publications, 31, (1997).

[22] V. V. Prasolov, The Lobachevsky geometry, MCCME, M, 2004.

[23] G. H. Hardy, E. M. Wright, An Introduction to the Theory of Numbers, Oxford University Press, 1975.

B.Verkin Institute for Low Temperature Physics and Engineering of the National Academy of Sciences of Ukraine, Kharkiv, 61103, Ukraine

E-mail address: aborisenk@gmail.com, suhdaria0109@gmail.com 